\title{
The Effects of Three Dimensional Structures on Cosmic-Ray Propagation and Interstellar Emissions
}

\author{
Guðlaugur Jóhannesson ${ }^{* a}$, Igor V. Moskalenko ${ }^{b}$, Elena Orlando ${ }^{b}$ Troy A. Porter $^{b}$, \\ and Andrew Strong ${ }^{c}$ \\ ${ }^{a}$ Science Institute, University of Iceland \\ ${ }^{b}$ Hansen Experimental Physics Laboratory and Kavli Institute for Particle Astrophysics and \\ Cosmology, Stanford University, Stanford, CA 94305, U.S.A. \\ ${ }^{c}$ Max-Planck-Institut für extraterrestrische Physik \\ E-mail: gudlaugu@hi.is, imosestanford.edu, eorlando@stanford.edu, \\ tporterestanford.edu, aws@mpe.mpg.de
}

\begin{abstract}
Interstellar emissions from radio to high-energy gamma rays (>100 MeV), arising from various interactions between CRs and the ISM, ISRF and magnetic field, are currently the best way to characterize the physics of CRs throughout the Milky Way. To properly utilize the high quality data of instruments such as the Fermi-LAT we need detailed modeling of these interstellar emissions. Here we report on our process of including three-dimensional (3D) structures in the distribution of interstellar gas and the CR source distribution available in the GALPROP code and discuss the effects on the modelled interstellar emissions.
\end{abstract}

The 34th International Cosmic Ray Conference,

30 July- 6 August, 2015

The Hague, The Netherlands

\footnotetext{
* Speaker.
} 


\section{Introduction}

It is now a well known fact that the Milky Way is a spiral-arm galaxy as evident from different tracer of both stars and the interstellar medium (ISM) (see e.g. [1]). The Milky Way also contains a bar like structure in the inner Galaxy and the distribution of $\mathrm{H}$ I gas shows sign of both warping and flaring in the outer Galaxy [2]. The study of the three dimensional (3D) distribution of the ISM in the Milky Way has mostly been done using line emission surveys, such as that of CO [3] and H I [4]. The Doppler shift of the line velocity is used in conjunction with a model for the rotation velocity (e.g. [5]) to create a 3D view of the distribution. This method works well for regions in the outer Galaxy but uncertainties in the rotation curve and limitations of the method hinder accurate determination in the inner Galaxy. Only recently have people started using large stellar surveys and dust extinction to map the distribution of dust in the Galaxy to a greater precision (e.g. [6]). This method provides a more robust view of the structure of the ISM but is currently limited to small regions of the Galaxy, both in terms of angular regions and also distance from the Sun. The dust method is also limited to probing the total amount of dust only and gives little information on the different phases of the ISM.

The distribution of cosmic-ray (CR) sources and the ISM in the Galaxy is one of the key ingredients to CR propagation models (e.g. [7]). These distributions play a crucial role when modeling the interstellar emissions arising from the interactions between CRs and the ISM and have also been shown to have a non-trivial effect on the interpretation of the observations of secondary CR spectra at earth (e.g. [8]). The incorporation of a detailed 3D model of the ISM, the interstellar radiation field (ISRF), the Galactic magnetic field, and the distribution of CR sources in CR propagation codes is therefore an important step when modeling the interstellar emissions to further our understanding of $\mathrm{CR}$ physics.

In this proceedings we describe our effort of modeling the 3D structure of the atomic and molecular gas components in the Milky Way. The focus of this work is to create a realistic 3D model of the whole Milky Way that can be used in CR propagation calculations. Our method of choice is using the line emission surveys and models for gas rotation, but instead of converting the line emission surveys, provided in the Galactic coordinates $(l, b, v)$ directly into a $(x, y, z)$ distribution we opt for the modeling approach. There are two main reasons for using this approach: The modeling method allows for a smooth interpolation over the Galactic center and Galactic anticenter where the relationship between gas-velocities and distance is very uncertain and the method also provides a smoother results that is less affected by the "fingers of God", the fictitious structures that seem to point along the line of sight from the Sun because of limited resolution allowed by the conversion from velocity to distance.

\section{The GALGAS code}

The GALGAS code is designed to take a model of the 3D number density distribution of gas in the Milky Way and turn it into the equivalent line intensity cube in Galactic coordinates $(l, b, v)$ given a model for the 3D velocity field in the Milky Way. The code can handle arbitrary shape for both the number density distributions and velocity field, although it has only been tested with a disk-like morphology for the number density and small perturbations to a circular rotation 
velocity field. The code has been fully parallelized with OpenMP and care has been taken to make it as efficient as possible. The current version is limited to optically thin line emission, but it can easily be extended to account for radiative transfer. To make the code as useful as possible we have designed it with extensibility in mind. Both the number density and velocity distributions are specified with an XML file where you can easily choose between several different building blocks and join them in different ways. It is also very easy to design and code your own building blocks that are then registered to the main code and can be used through the XML file interface.

The main purpose of this package is to improve upon the current cylindrically symmetric $\mathrm{H} \mathrm{I}$ and $\mathrm{H}_{2}$ gas distributions $[10,11,12]$ currently available in the GALPROP ${ }^{1}$ package [9]. We will hereafter refer to these as the current model. To do that we parametrize our building blocks and optimize the parameter values in a fit to survey data. We currently compare the models to data using a $\chi^{2}$ function using the Gaussian noise of the line emission survey to weight each point, but this can easily be extended. The parameters can be optimized with either the Minuit2 package from ROOT ${ }^{2}$ or the MultiNest ${ }^{3}$ package [13]. The code can merge survey data from different experiments and all points are re-binned to a common grid that is user specified. It is also possible to filter out specific regions of the sky in $(l, b, v)$ space, such as external galaxies in the survey, and to specify regions where the velocity information is ignored. The latter is very useful around the Galactic center and anti-center where the velocity provides little information on distance.

\section{Example Models}

To test the code we decided to fit a simple two-dimensional (2D) azimuthally symmetric model to the $\mathrm{CO}$ line survey of [3]. The radial number density distribution was modeled on a spatial grid with points at $0,1,2,3,4,6,8,10,20$, and $50 \mathrm{kpc}$ interpolated with a cubic spline, where the number density was free to vary at each grid point. The disk scale height was also modeled with a cubic spline between the grid points at the same radial distance. The velocity field was chosen to be a simple cylindrical rotation around the Galactic center with the rotation curve of [5]. Velocity information was ignored for regions with $-10^{\circ}<l<10^{\circ}$ and $170^{\circ}<l<190^{\circ}$. The parameters of the model were tuned with the Minuit2 VariableMetric minimizer. We note that the models here are examples only, we are still working on the final functional forms for our model that best describe the data.

The radial profiles for both the scale height and the number density distribution is displayed in figure 1 along with the current model. As we can see, the current model and our new model show some similar characteristics. There is a pronounced peak at the Galactic center and an enhancement between roughly 4 and $8 \mathrm{kpc}$. The new model, however, has a factor of 2 to 3 higher number density between 4 and $8 \mathrm{kpc}$ while it is much smaller between 1 and $3 \mathrm{kpc}$ and beyond $9 \mathrm{kpc}$. The scale height of the new model is also systematically lower in most radial ranges where it matters. We note that these results are preliminary and the exact profiles depend on our choice for both the radial velocity and the functional form chosen for the radial distributions and the vertical scale. Figure 2 shows the radial profiles from an analysis where instead of using the smooth model from [5] for

\footnotetext{
${ }^{1} \mathrm{http}: / /$ galprop.stanford.edu

${ }^{2}$ http://root.cern.ch

${ }^{3}$ https://ccpforge.cse.rl.ac.uk/gf/project/multinest/
} 


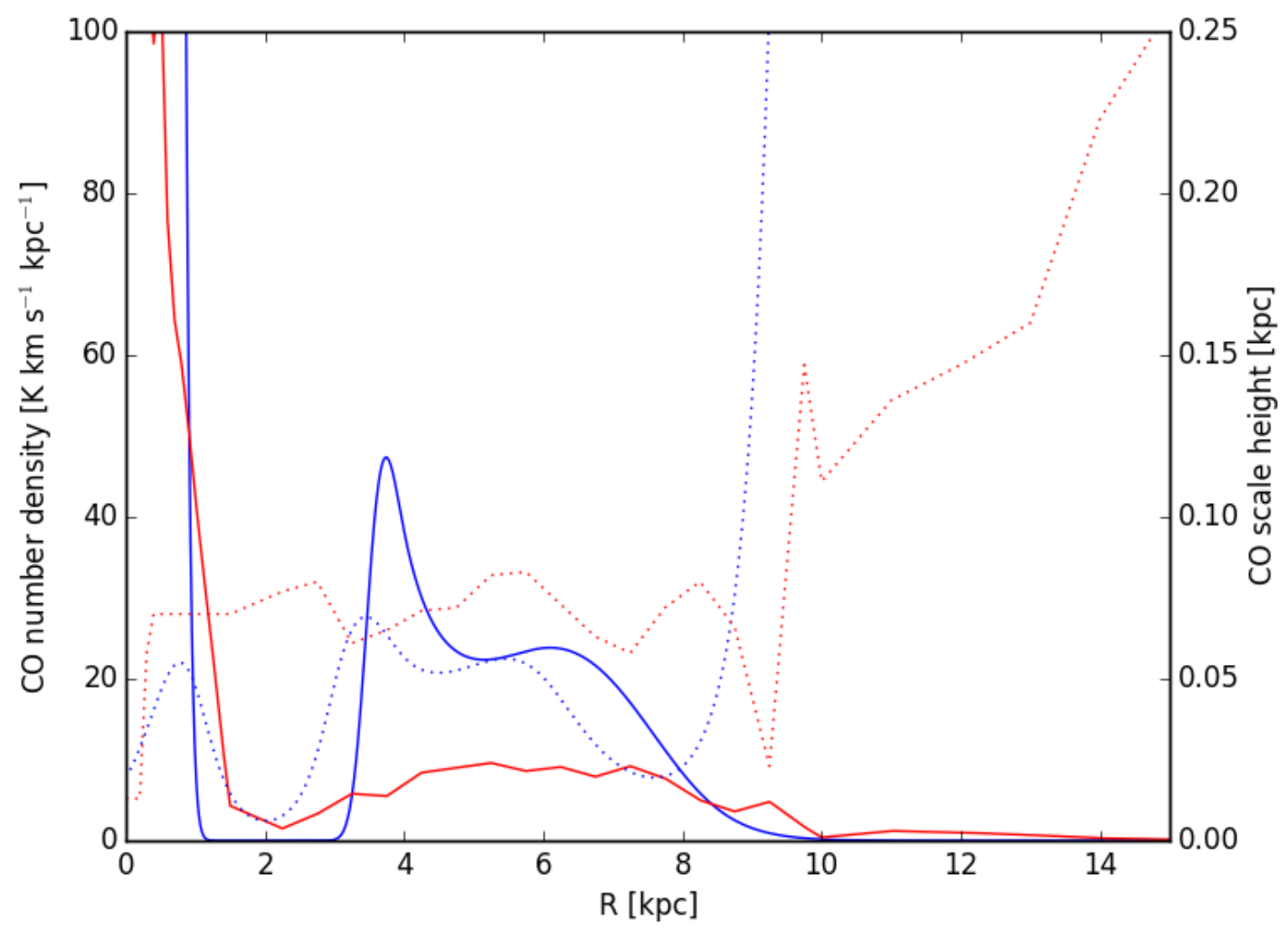

Figure 1: Radial number density distributions (solid curves) and their respective scale heights (dotted curves) for the current model (red curves) and our new model (blue curves) found using GALGAS code on CO data and the rotation curve from [5]. The large scale height of the new model beyond the solar location is likely fictitious. The model density goes rapidly to zero and there is little constraining power for the scale height.

our rotation curve we use the data from [14] with linear interpolation. The main characteristics stay the same while the details can differ quite a bit.

Another important factor is the resolution used when performing the $\chi^{2}$ fit. The current results are based on a resolution of around $0^{\circ} .25$ and degrading the resolution to $0^{\circ} .5$ or even $1^{\circ}$ resulted in a larger number density at the Galactic center but smaller between 4 and $8 \mathrm{kpc}$. These lower resolution results are therefore much closer to the current models than the ones we show in the figures. We are currently exploring and documenting several of these systematic effects.

\section{Looking ahead}

The main goal of this work is to constrain the 3D structure of the Galaxy, including the location and density structure of the major spiral arms in our Galaxy. This is a non-trivial work, because the number of parameters in the model rapidly increase as more structure is added. Each of the two radial distributions in the example disk-like models shown above had 10 parameters, totaling 20 parameters for the entire model. Trying to do the same with four additional spiral arms would 


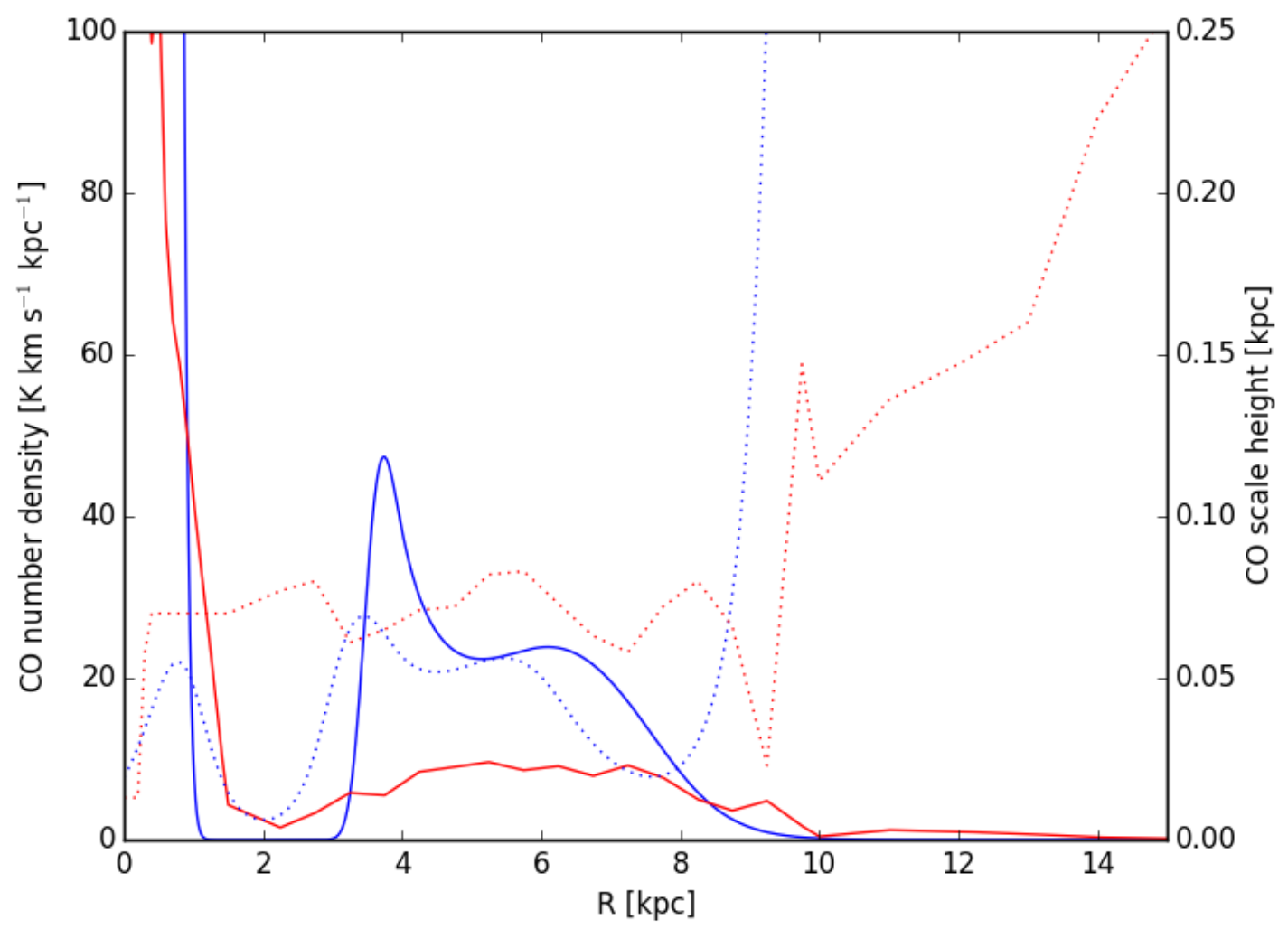

Figure 2: Similar to figure 1 except the analysis used a linear interpolation between the data points in [14] to model the rotational velocity of the gas.

lead to a model with over 100 parameters, creating a very difficult minimization problem. Each additional component also adds to the complexity of the model and the time it takes to perform each $\chi^{2}$ evaluation. Reducing the number of parameters and the complexity of the model is therefore one of the main goals in this effort. We are currently exploring several avenues towards this goal.

As a demonstration for the possible magnitude of the effects of 3D structure on CR propagation we performed GALPROP calculations using a mock spiral arm model based on the information in [1]. We put all the CR sources and half of the interstellar gas into the spiral arm structure and compared it to models with an azimuthally symmetric distribution. Figure 3 shows the ratio of the total $\gamma$-ray skymaps at $10 \mathrm{GeV}$ from both analyses. The skymap clearly shows the asymmetric structures associated with the spiral arms and illustrates the magnitude of the effect. This simple change in the azimuthal distribution (not radial) of the CR sources and gas can change the resulting CR emission maps by more than 50 percent in a way that cannot be compensated for by changing the radial profile only. This model also changes the predicted primary to secondary ratio of B/C at all energies in a subtly energy dependent way while the change in the spectrum of protons was much smaller and mostly below $1 \mathrm{GeV}$ due to the change in ionization energy losses. The variations between the mock spiral arm and the azimuthally symmetric models are significant and clearly show the importance of getting a correct description for the 3D structure of the ISM when 


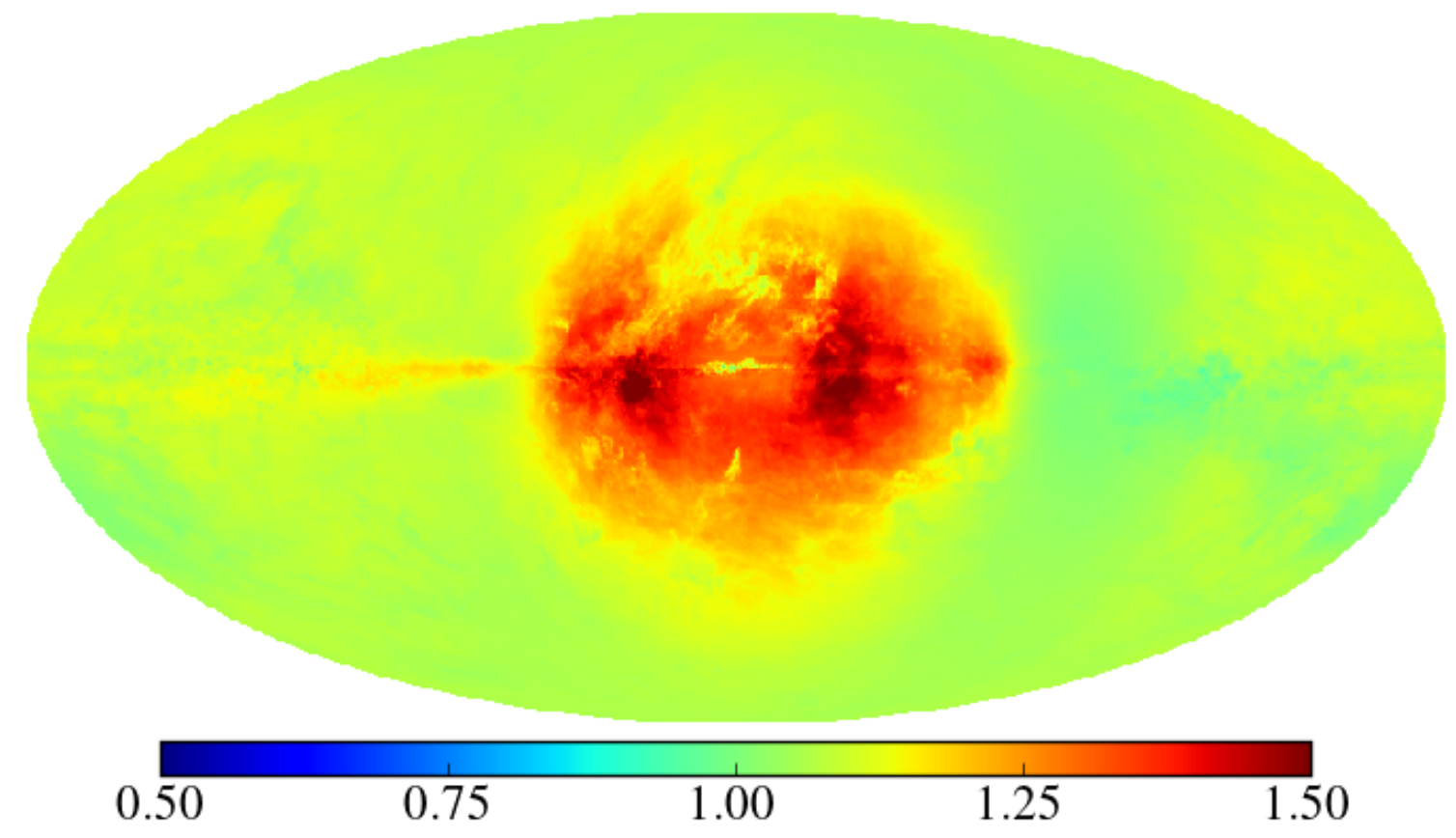

Figure 3: Ratio of the total $\gamma$-ray skymaps at $10 \mathrm{GeV}$ calculated with GALPROP for two ISM gas and CR source models: a model incorporating the spiral arm structure in the gas and CR source distributions vs. a standard model with cylindrical symmetry. The enhanced emission at the spiral arm tangents are visible in the map.

modeling CR related processes in the Milky Way.

\section{Acknowledgments}

GALPROP development is supported through NASA Grants No. NNX10AE78G, NNX13AH72G, and NNX13AC47G.

\section{References}

[1] J.P. Vallee, Catalog of Observed Tangents to the Spiral Arms in the Milky Way Galaxy, ApJS 2014 (215) 9 [arXiv:1409.4801]

[2] P.M.W. Kalberla and J. Kerp, The H I Distribution of the Milky Way, ARA\&A 2009 (47) 27

[3] T.M. Dame, D. Hartmann, and P. Thaddeus, The Milky Way in Molecular Clouds: A New Complete CO Survey, ApJ 2001 (547) 792 [astro-ph/0009217]

[4] P.M.W. Kalberla et al., The Leiden/Argentine/Bonn (LAB) Survey of Galactic HI. Final data release of the combined LDS and IAR surveys with improved stray-radiation corrections, A\&A 2005 (440) 775 [astro-ph/0504140]

[5] Y. Sofue, M. Honma, and T. Omodaka, Unified Rotation Curve of the Galaxy - Decomposition into de Vaucouleurs Bulge, Disk, Dark Halo, and the 9-kpc Rotation Dip -, PASJ 2009 (61) 227 [arXiv:0811.0859] 
[6] M. Schultheis et al., Extinction Maps toward the Milky Way Bulge: Two-dimensional and Three-dimensional Tests with APOGEE, AJ 2014 (148) 10 [arXiv:1405.2180]

[7] M. Ackermann et al., Fermi-LAT Observations of the Diffuse $\gamma$-Ray Emission: Implications for Cosmic Rays and the Interstellar Medium, ApJ 2012 (750) 35 [arXiv:1202.4039]

[8] G. Johannesson, I.V. Moskalenko, and T.A. Porter, Toward 3D mapping of the interstellar medium in the Milky Way: impact on cosmic rays and diffuse emission, in proceedings of ICRC 2013.

[9] A.E. Vladimirov et al., GALPROP WebRun: An internet-based service for calculating galactic cosmic ray propagation and associated photon emissions, Computer Physics Communications 2011 (182) 1156 [arXiv:1008.3642]

[10] L. Bronfmann, R.S. Cohen, H. Alvares, J. May, and P. Thaddeus, A CO survey of the southern Milky Way - The mean radial distribution of molecular clouds within the solar circle, ApJ 1988 (324) 248

[11] J.G.A. Wouterloot, J. Brand, W.B. Burton, and K.K. Kwee, IRAS sources beyond the solar circle. II Distribution in the Galactic warp, A\&A 1990 (230) 21

[12] K. Ferrière, W. Gillard, and P. Jean, Spatial distribution of interstellar gas in the innermost 3 kpc of our galaxy, A\&A 2007 (467) 611

[13] F. Feroz, M.P. Hobson, and M. Bridges, MULTINEST: an efficient and robust Bayesian inference tool for cosmology and particle physics, MNRAS 2009 (398) 1601 [arXiv:0809.3437]

[14] Y. Sofue, Grand Rotation Curve and Dark Matter Halo in the Milky Way Galaxy, PASJ 2012 (64) 8 [arXiv:1110.4431] 